\title{
An analytic Study of the Key Factors Influencing the Design and Routing Techniques of a Wireless Sensor Network
}

\author{
Yogita Bahuguna, Deepak Punetha, Pooja Verma \\ Department of Electronics \& Communication Engineering, Tula’s Institute, Dehradun, India
}

\begin{abstract}
A wireless sensor network contains various nodes having certain sensing, processing \& communication capabilities. Actually they are multifunctional battery operated nodes called motes. These motes are small in size \& battery constrained. They are operated by a power source. A wireless sensor network consists of a huge number of tiny sensor nodes which are deployed either randomly or according to some predefined distribution. The sensors nodes in a sensor network are cooperative among themselves having self-organizing ability. This ensures that a wireless network serves a wide variety of applications. Few of them are weather monitoring, health, security \& military etc. As their applications are wide, this requires that sensors in a sensor network must play their role very efficiently. But, as discussed above, the sensor nodes have energy limitation. This limitation leads failure of nodes after certain round of communication. So, a sensor network suffers with sensors having energy limitations. Beside this, sensor nodes in a sensor network must fulfill connectivity \& coverage requirements. In this paper, we have discussed various issues affecting the design of a wireless sensor network. This provides the readers various research issues in designing a wireless sensor network.
\end{abstract}

Keywords - Wireless Sensor Network, Nodes Energy, QoS, Connectivity.

\section{Wireless Sensor Network}

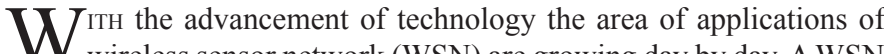
wireless sensor network (WSN) are growing day by day. A WSN contains many sensor nodes of the order of hundreds or even thousands. They are tiny in size having limited energy and communication range. Besides this, they suffer from limited sensing, computational and transmission capabilities. In a wireless network information is transferred from a source (sensor nodes) to a sink (base station). The base station can be placed inside or outside the monitoring area. The sensor nodes are scattered over a huge geographical area randomly [13] [14]. The nodes in a wireless network can either communicate among themselves or to a base station. These nodes are sophisticated having intra and inter communication capabilities. In a wireless network the nodes play different roles as per the necessity of communication. A node can provide an interfacing between sources and sink thereby reducing the energy consumption by providing an energy efficient path to route the data from the source to the sink [11]. These nodes are called gateways. Gateway nodes selection is essential to prevent the death of the nodes due to excessive energy consumption. Relay nodes (routers) are used to expand the coverage area and to provide backup routes in case of failure of nodes and data traffic.

A leaf node (endpoint) is used to establish an interfacing between a wireless network and a sensor that is wired to it. Actuators are the sensors used to interact with the physical environment which has to be

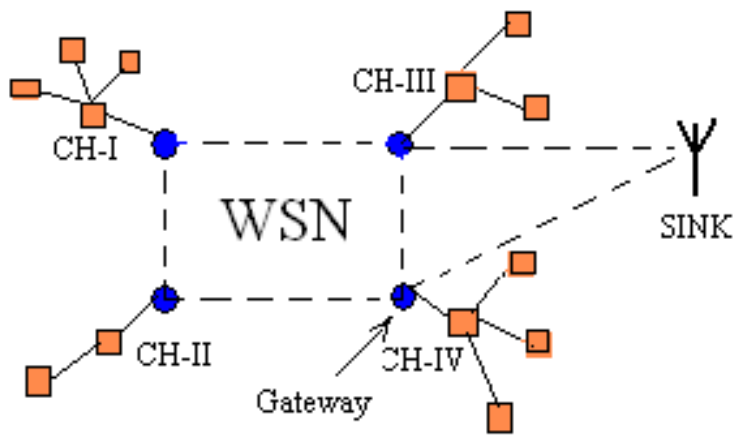

Fig. 1. A basic block diagram of a WSN

controlled. The vital applications of wireless networks are enormous. This section depicts various areas of application of WSNs [8]. They serve the different purpose starting from home applications, health applications and military applications to the environmental applications. In the environmental applications they are used to monitor a wide range of ambient conditions such as temperature, humidity, pressure, noise level and soil make up etc which affect the crops and live stocks [24]. Moreover, in forest fire and flood detection, earth monitoring and pollution study the use of wireless network is crucial. The wireless networks are being used in health sector efficiently in various fields. To monitor doctor and patient inside a hospital, to monitor the activities in a hospital, to monitor the activities and processes in tiny insects are few of them [17]. Beside these, sensors can be deployed in a health care unit to control drugs administration, thus ensuring the safe and the better drug services to the patients. WSNs are playing an unavoidable role in home applications as well. Several home appliances such as microwave ovens, vacuum cleaners, refrigerators are now equipped with sensors, thus enabling these appliances to interact with one another or to an external link via the internet and finally offering better services to the

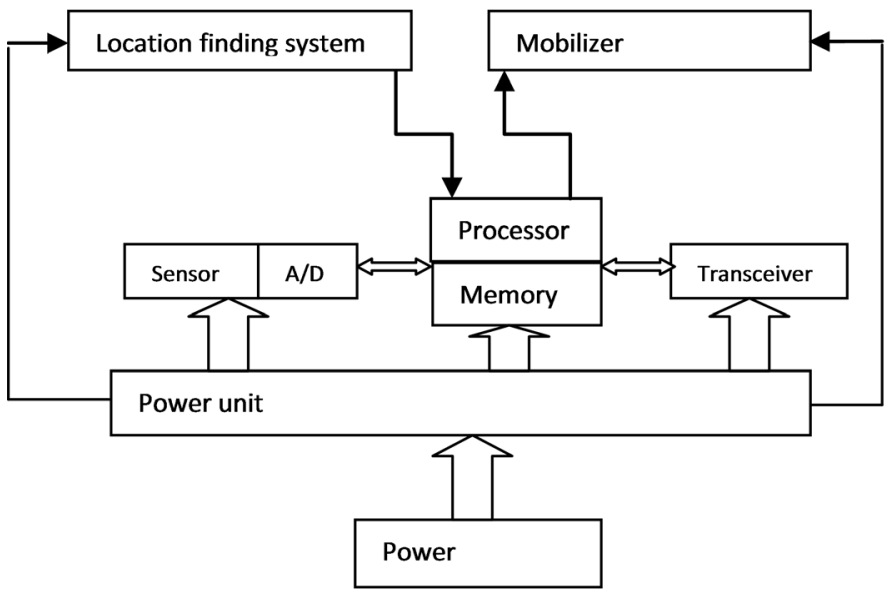

Fig. 2. A block diagram of a WSN. 
end user [10] [16]. Last but not the least, the role of WSNs in military application is pervasive. Sensor nodes are deployed in remote places where human interference except military is not possible [23]. They are used to monitor intrusion of terrorists, equipment, vehicle etc from the outside. Wireless sensors can be used to gather data of battlefield [12] [22] [25]. In all the applications the role of sensor node is crucial. Therefore, remedies have to take in order to minimize decay of nodes, so as to prolong the network lifetime.

\section{Network Structure}

A sensor node comprises of four main units namely a sensing unit, a processing unit, a transceiver unit and a power unit (fig.1). The sensing unit can be further divided into two parts: sensors and an analog to digital converter [13] [15]. The function of the sensor is to first sense the physical phenomenon.

Then it converts the sensed physical phenomenon into an analog signal. The output of the sensor is then fed to an analog to digital converter circuitry which converts an analog signal into digital signal appropriate for further transmission. The output of the sensing unit is then applied to the processing unit which generally consists of a processor and a storage device. The function of a processor is to generate necessary instructions and commands in order to facilitate the coordination among the sensors node [4]. There by performing the sensing task. The third unit is transceiver which may be a passive or active optical device [20]. The term transceiver is used for the transmitter and receiver combined, which is equipped with the ability of transmitting and receiving a signal. The transceiver unit of sensor nodes connects the node to the network. The fourth unit is a power unit driven by a power generator such as a solar cell which uses energy scavenging techniques and extracts energy from the environment. Energy scavenging is very important since sensor nodes may remain un- attendant in a remote place for several months or years without human interference [18]. Beside these four, two sub-units are a location finding system and a mobilizer. The location finding system

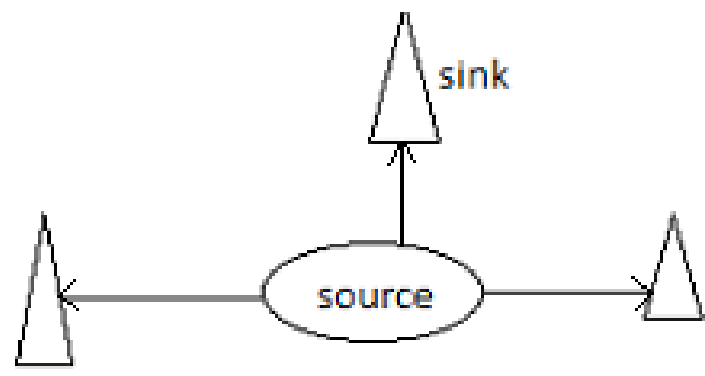

Fig. 3.1 Single Hop Communication.

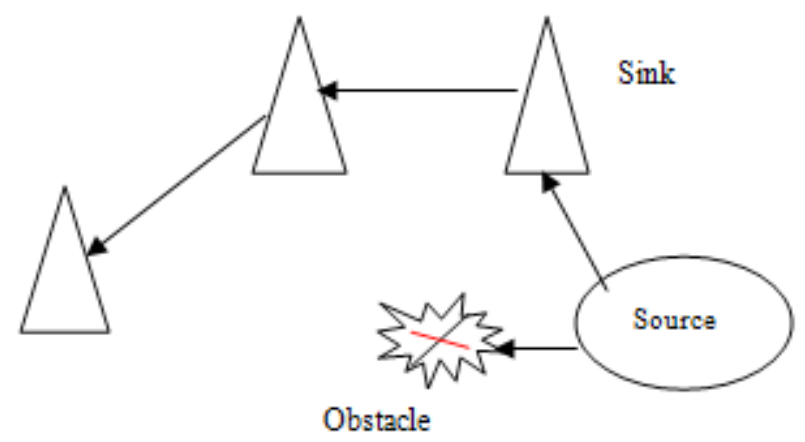

Fig. 3.2 Multi Hop Communication is necessary in a WSN to apply routing techniques in a proper way. The routing techniques use the information about the sensor's location provided by the location finding system. A mobilize is optional which is needed in case of mobile sensor nodes.

A WSN can be of two types depending upon the manner of data transmission viz. single hop and multi hop WSN. In case of single hop transmission (fig.2.1) data is transferred from gateway to the sensor node whereas in case of multi hop communication (fig.2.2) two or more sensor nodes are used in data path. In multi hop network, the data is transferred from one node to another in case obstacle like hills, mountains, lakes etc is found in the data path. Many routing techniques are based on multi hop networking of WSNs.

\section{Problem Definition}

As discussed in the earlier sections that sensor nodes are energy constraints operated by power source like solar cells. These sensor nodes are deployed randomly or according to some predefined criterion. But in general deployment of sensor nodes can be done randomly over a region of interest by dropping them from the helicopter or manually [21]. These nodes have self-organizing capability and they gather the information from the event under consideration, transmit the collected information to the sink via neighbor nodes. They are used in several applications like health monitoring, environment monitoring, home monitoring and military applications where the role of sensor node is most important. But sensor nodes are limited in terms of energy and range of communication, therefore careful deployment strategies and design parameters have to be considered when using WSNs in the above applications. In the past years many factors that affect the design of WSNs have been elaborated and different routing techniques based on minimizing the power utilization by the sensor nodes came into existence. In this section we give an overview of the issues that influence the design of WSNs and that must be overcome in order to facilitate effective communication.

\section{A. Nodes deployment}

The manner in which deployment of sensor nodes is done greatly affects the design of sensor network as well as routing techniques. As discussed in the previous sections that the deployment of sensor nodes could be done either in a random fashion or according to predefined set of rules (fig.3). Whatever is the way of deployment, the number of sensor nodes should be well enough to cover the whole geographical area. The density of sensor nodes in a geographical area of $1 \mathrm{~m} 3$ can be as high as 20 nodes [19]. However the distribution of sensor nodes depends on the need of application. In

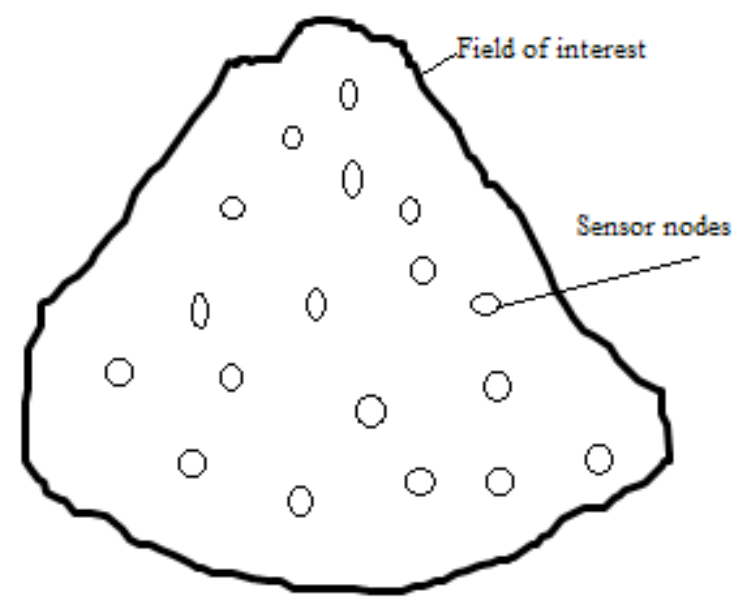

Fig. 4. Nodes Deployed in a Field of Interest. 
the deployment phase, the topology that minimizes the installation cost while maximizing the flexibility should be adopted. Failure of the sensor nodes is common in the network; therefore the need of introducing the new sensors arises in the existing network. The deployment topology used should be flexible to manage with the introduction of new sensor nodes. Beside this, the topology adopted should be able to prop up the self-organizing quality of the sensor node in a network.

\section{B. Power consumption}

In WSNs many routing protocols have been proposed. They mainly consider the power requirements of a sensor network. This implies energy limitations of a sensor network have great influence on the routing techniques. The sensor nodes are multifunctional; they work as a sensor node and as gateways in the data path [4-5] [9]. Therefore for a WSN to work properly it becomes important that its sensor can conserve their energy so that network lifetime can be prolonged. If by chance a sensor failure occurs due to energy loss, it should not have an effect on the whole WSN. Usually multipath routing is adopted to transmit the data from the source to the sink (base station). Although single hop routing can also be applied to a WSN at the cost of more power consumption.

\section{Mobility of sensor node}

Sensor nodes are one of the most important components of a wireless sensor network [6]. A sensor node plays multifunctional roles viz. a data router or a gateway. Many applications may require static behavior of sensor nodes. Whereas, some application may need sensor nodes to behave dynamically. This necessitates the design of wireless network to be able to facilitate the mobility of sensor node.

\section{Fault tolerance}

The sensor network consists of a huge number of tiny sensors which are prone to frequent failures due to various reasons. Since they are battery operated, therefore insufficiency of power is one of the main causes of nodes death [5] [11]. Other reasons may include disturbances from the environment and physical damage. Due to mentioned faults if any of the nodes fail, it should not influence the overall functioning of the sensor network. Therefore, a WSN should be well reliable or trust worthy to cope up with the disturbances arising due to nodes death. Thereby smoothening the overall performance of the sensor network and sustaining the networks life. The Poisson's distribution provides the mathematical expression for the reliability of a sensor node $\mathrm{K}$ of not having a failure within a time interval $(0, t)$;

$$
R k(t)=\exp (-\lambda k t)
$$

Where $R_{k}(t)$ is the reliability of a $K^{\text {th }}$ sensor node, $\lambda_{k}$ is the failure rate of sensor node $\mathrm{K}$ and $\mathrm{t}$ is the time period

\section{E. Security}

With the advancement of new technologies the areas of application of WSNs have grown surprisingly [6]. There use in mission critical operations such as battlefield surveillance, intrusion detection and target monitoring etc. creates the concern on security issues. In case of a mobile sink or gateways security is a crucial issue to be considered when designing wireless sensor network and routing protocol. Moreover, the wireless nature of the network, dense deployment of nodes, resource limitations of nodes and unavailability of fixed infrastructure creates a big concern on the network security. The wireless networks are more prone to attacks than wired networks. The design of a wireless network should be flexible in the sense that security level is changed when there is a change in available resources.

\section{F. Communication energy}

The main objective of a wireless sensor network is data communication which involves both data transmission and data reception [7]. Data is transferred from the sensor node to the base station via some gateways. As the number of data transmissions is increased, more energy will be devoted in the communication process.

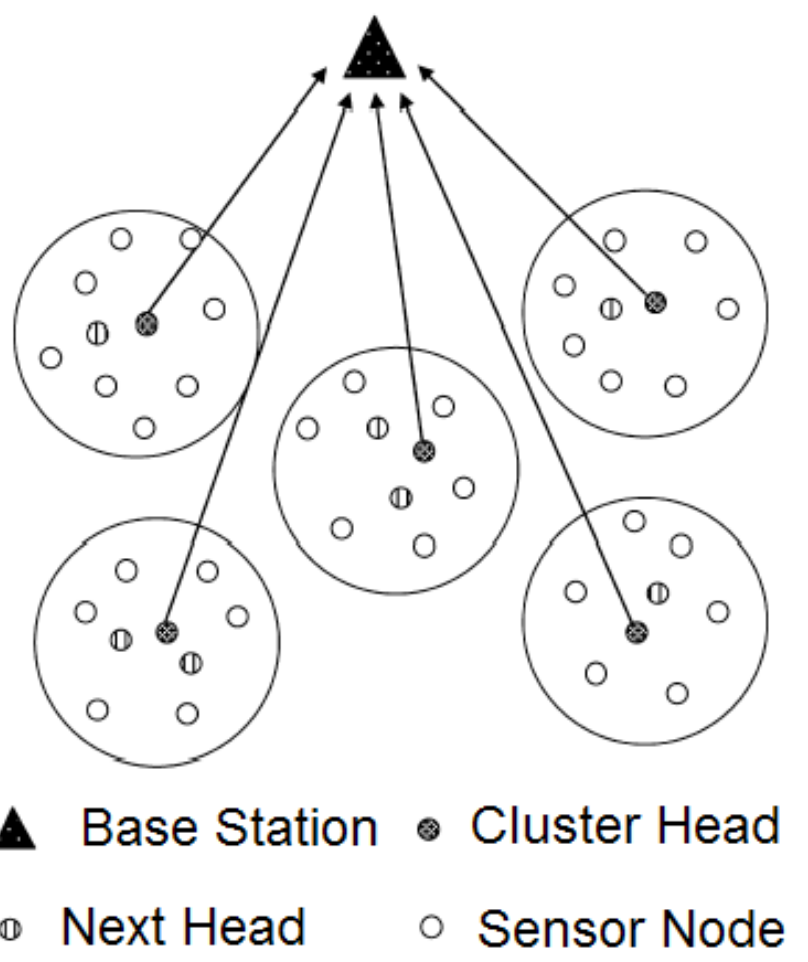

Fig. 5. Cluster formation.

Therefore to lessen the number of transmissions from the base station to the sink nodes, clustering is viable.

\section{Simulation AND EXPERIMENT RESUlts}

The simulation result has been done by MATLAB tool. ECSSCoM (Energy-aware Clustering Sensor Scheduling Coverage Maintenance) a coverage maintenance protocol for wireless sensor networks. This protocol uses two techniques namely network clustering and sensor

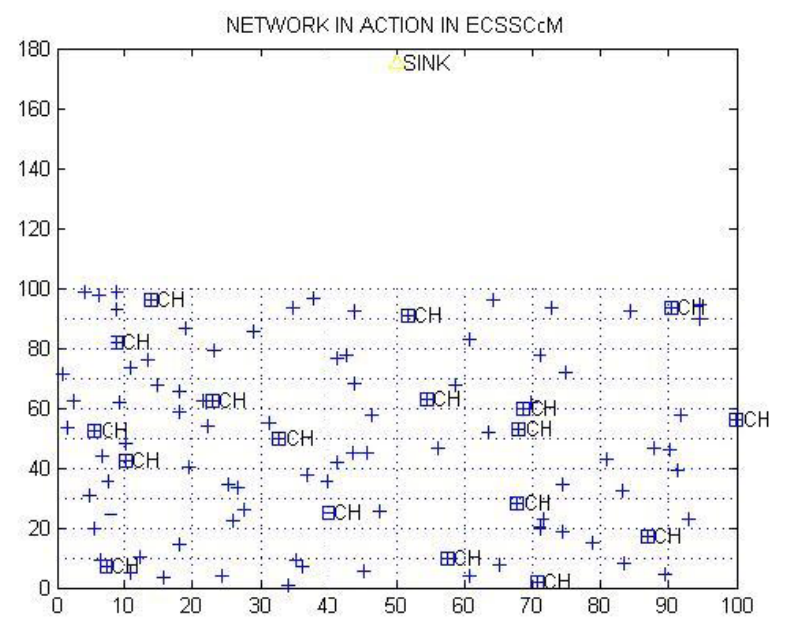

Fig. 6.1 Node deployment in ECSSCoM. 


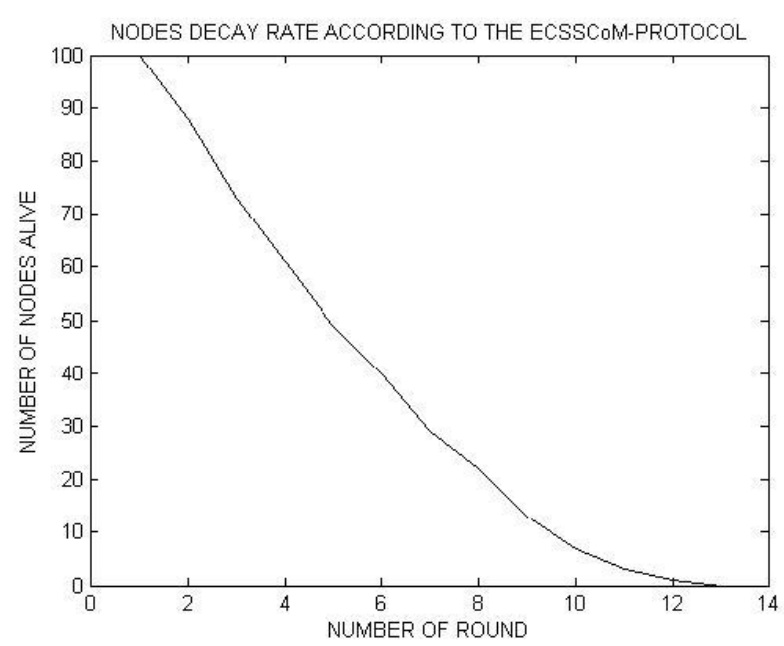

Fig. 6.2 Performance of nodes in ECSSCoM.

activity scheduling.

The simulation has been categories in several platform in which simulation of ECSSCoM has been performed as well as simulation of ECSSCoM and improved ECSSCoM with object consideration taken place.

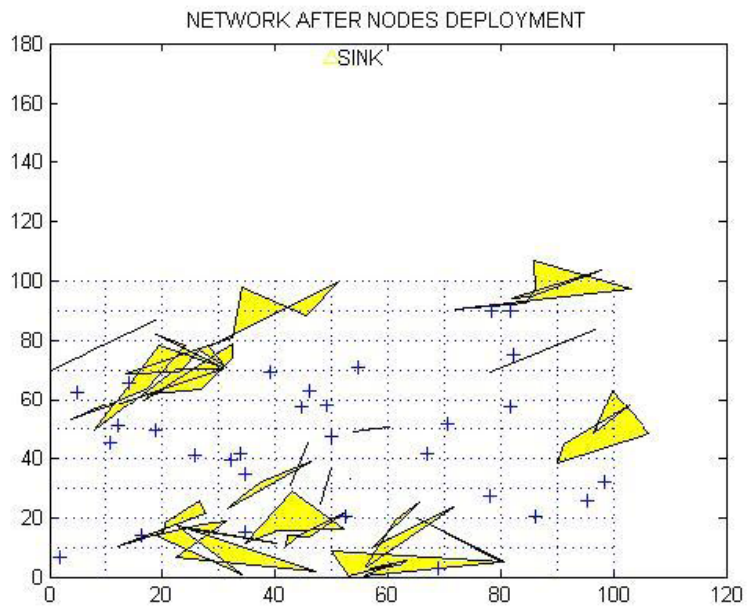

Fig. 7.1 Node deployment in ECSSCoM with obstacle consideration

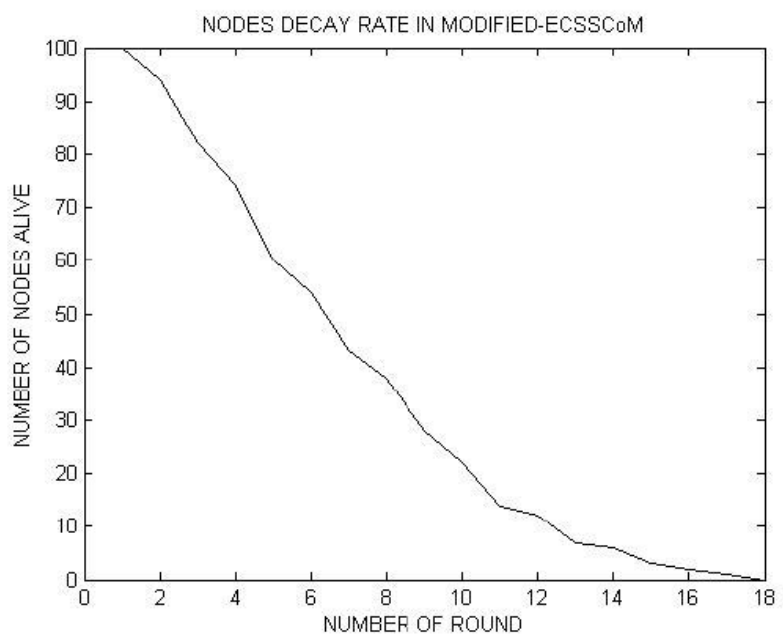

Fig. 7.2 Performance of nodes in ECSSCoM with obstacle.
The analysis and simulation results have been obtained for ECSSCoM for 100 nodes with energy of 2 Joule. In this execution it has been observed that every nodes of the WSN first find the neighboring nodes after that it form a cluster head.

\section{Conclusion}

In this paper, a comprehensive study has been executed over various key parameter like nodes deployment, mobility of sensor nodes, communication energy and power consumption etc. The parameters discussed in this manuscript play an imperative role in designing the wireless sensor network simultaneously providing a platform for new research areas on designing and routing techniques for WSNs by pinpointing the various challenges.

\section{REFERENCES}

[1] V.B. Semwal, V.B. Semwal, M. Sati and S. Verma, "Accurate location estimation of moving object in Wireless Sensor network," International Journal of Interactive Multimedia and Artificial Intelligence, Vol. 1, No. 4,pp. 71-75, 2011.

[2] García, Óscar, Ricardo S. Alonso, Dante I. Tapia, and Fabio Guevara, "Wireless Sensor Networks and Real-Time Locating Systems to Fight against Maritime Piracy," International Journal of Interactive Multimedia and Artificial Intelligence, Vol. 1, no. 5, pp. 14-21, 2012.

[3] Yogita Bahuguna, Jyoti Rawat, "An Efficient Routing Protocol in Heterogeneous Wireless Sensor Network Considering Obstacle," IEEE International Conference on Advances in Computing \& Communication Engineering (ICACCE-2015), Dehradun, India, pp: 249 - 252, 1-2 May 2015.

[4] A. Bakre, B.R. Badrinath, I-TCP: indirect TCP for mobile hosts, Proceedings of the 15th International Conference on Distributed Computing Systems, Vancouver, BC, May 1995, pp. 136-143.

[5] A. Cerpa, D. Estrin, ASCENT: adaptive self-configuring sensor networks topologies, UCLA Computer Science Department Technical Report UCLA/CSDTR-01-0009, May 2001.

[6] A. Cerpa, J. Elson, M. Hamilton, J. Zhao, Habitat monitoring: application driver for wireless communications technology, ACM SIGCOMM'2000, Costa Rica, April 2001.

[7] A. Chandrakasan, R. Amirtharajah, S. Cho, J. Goodman, G. Konduri, J. Kulik, W. Rabiner, A. Wang, Design considerations for distributed microsensor systems, Proceedings of the IEEE 1999 Custom Integrated Circuits Conference, San Diego, CA, May 1999, pp. 279-286.

[8] A. Gallais and J. Carle, "An Adaptive Localized Algorithm for Multiple Sensor Area Coverage", IEEE 21st International Conference on Advanced Information Networking and Applications (AINA 2007), Niagara Falls, Canada, May 2007.

[9] B.G. Celler et al., An instrumentation system for the remote monitoring of changes in functional health status of the elderly, International Conference IEEE-EMBS, New York, 1994, pp. 908-909.

[10] B. Wang, C. Fu, and H. B. Lim, "Layered Diffusion-based Coverage Control in wireless sensor networks," Computer Networks Journal, Vol. 53, No. 7, pp. 1114-1124, 2009.

[11] Goyal, D.,Tripathy, M.R. "Routing Protocols in Wireless Sensor Networks: A Survey," IEEE 2nd International Conference on Advanced Computing \& Communication Technologies (ACCT), Rohtak, Haryana, 7-8Jan.2012.

[12] G.D. Abowd, J.P.G. Sterbenz, Final report on the interagency workshop on research issues for smart environments, IEEE Personal Communications (October 2000) 36-40.

[13] I-F.Akyildiz, W. Su,Y.Sankarasubramaniam, and E. Cayirci, "Wireless sensor networks: a survey," Computer Networks, Vol. 38, No. 4, pp 393422, 2002.

[14] I.F. Akyildiz, W. Su, A power aware enhanced routing (PAER) protocol for sensor networks, Georgia Tech Technical Report, January 2002, submitted for publication.

[15] J. Agre, L. Clare, An integrated architecture for cooperative sensing networks, IEEE Computer Magazine (May 2000) 106-108.

[16] Khanouche, M.E., Ouada,F.S.,Ouguigui,S., "Energy-Aware clustering and sensor shduleding coverage maintence for wireless sensor networks," IEEE 
International Conferenece on Green Computing and Communications (GreenCom), Besancon, 20-23 Nov.2012 .

[17] Laiali Almazaydeh, Eman Abdelfattah, Manal Al- Bzoor, and Amer AlRahayfeh, "Performance Evaluation Of Routing Protocols In Wireless Sensor Networks," International Journal of Computer Science and Information Technology, Volume 2, Number 2, April 2010.

[18] M. Bhardwaj, T. Garnett, A.P. Chandrakasan, Upper bounds on the lifetime of sensor networks, IEEE International Conference on Communications ICC'01, Helsinki, Finland, June 2001.

[19] N. Bulusu, D. Estrin, L. Girod, J. Heidemann, Scalable coordination for wireless sensor networks: self-configuring localization systems, International Symposium on Communication Theory and Applications (ISCTA 2001), Ambleside, UK, July 2001.

[20] P. Bauer, M. Sichitiu, R. Istepanian, K. Premaratne, The mobile patient: wireless distributed sensor networks for patient monitoring and care, Proceedings 2000 IEEE EMBS International Conference on Information Technology Applications in Biomedicine, 2000, pp. 17-21.

[21] P. Bonnet, J. Gehrke, P. Seshadri, Querying the physical world, IEEE Personal Communications (October 2000) 10-15.

[22] V. Tran-Quang, T. Miyoshi, "A novel gossip-based sensing coverage algorithm for dense wireless sensor networks," Comput. Netw. 53 (2009), pp. 2275-2287, September 2009.

[23] Vinh Iran Quang,Miyoshi T., "An Algorithm for Sensing Coverage Problem in Wireless Sensor Networks," 2008 IEEE sarnoff Symposium ,Princeton,New Jersey,USA,Paper No. S3.5,April 2008.

[24] Xin Liu, "Coverage with Connectivity in Wireless Sensor Networks",3rd international conference on broadband communications,networks and systems,2006,San jose,CA.

[25] Z. Liu, Q. Zheng, L. Xu, and X. Guan, "A distributed energy efficient clustering algorithm with improved coverage in wireless sensor networks," Future Generation Computer Systems Journal, Vol.28, No. 5, pp. 780-790, 2012.

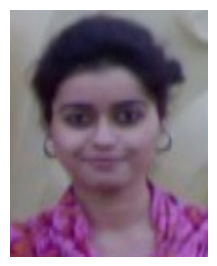

Ms. Yogita Bahuguna obtained her B.Tech degree in AIE from DIT, Dehradun (UPTU). She received her M. TECH degree from UTU, Dehradun in Digital communication. Currently she is serving Tula's institute, Dehradun as an assistant professor in ECE department. She has an experience of more than 6.5 years in teaching and one year industry experience. She has worked for various organizations such as DIXON technologies pvt Ltd Noida, Alpine college, GRD IMT and Tula's institute, Dehradun. She has published various research papers in international conferences and journals. Her research area is Wireless Sensor Networks, Embedded System and Digital Communication.

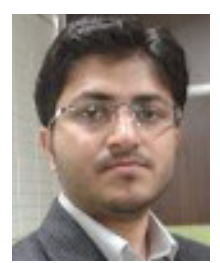

Mr. Deepak Punetha is serving Tula's Institute, Dehradun (Uttarakhand Technical University) as an Assistant Professor in Electronics \& Communication Engineering department. He has an experience of more than 5.5 years in teaching and research (Including research experience in CDAC, Mohali). He has completed his B.Tech in ECE from Dehradun Institute of Technology and M.E. (8.5 CGPA) in EPDT from PEC University of Technology, Chandigarh. $\mathrm{He}$ has worked for various organizations, such as Chitkara University, Himachal Pradesh (as AP), PEC University of Technology, Chandigarh (as Teaching Assistant), CDAC Mohali and Tula's Institute, Dehradun. He has published more than 35 research papers (included IEEE, Springer, Elsevier etc.) in reputed Conferences and International Journals. He is the member of reviewing committee of IEEE Explorer, Elsevier Science \& Technology, Springer (Journal of Intelligent \& Robotics Systems), Hindawi and various National and International Conferences and Journals. He has served as TPC member for many National \& International Conferences in the region. He is also an active member of different National and International Association of Electronics and Communication Engineers and Editorial Boards. His area of interest is Electronics Product Design and Technology, Face Recognition and Compression, Radiation Pattern analysis of different Antennas, Navigation and Emergency Alerting System, Robotics and Embedded Systems.

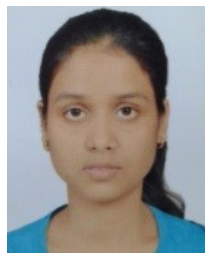

Ms. Pooja Verma completed her B.Tech. in Electronics \& Communication Engineering from N.I.E.T. Greater Noida India in 2012 and received her M.Tech degree in VLSI Design from Indira Gandhi Delhi Technical University for Women, Delhi. Currently she is serving Tula's institute, Dehradun as an assistant professor in ECE department. She has worked with C-DOT, Mehrauli and CSIRCSIO Chandigarh as trainee. She has published various research papers in international conferences and journals. Her interest includes VERILOG FPGA Design, low power VLSI Design. 